\title{
Singular equivalences arising from Morita rings
}

\author{
Nan Gao and Wen-Hui Zhao
}

\begin{abstract}
We obtain new classes of singular equivalences which are constructed from Gorenstein-projective modules.
\end{abstract}

\section{Introduction}

The singularity category $D_{s g}(A)$ of an algebra $A$ over a field $k$, introduced by R.O. Buchweitz in [4], is defined as the Verdier quotient $D_{s g}(A)=D^{b}(A$-mod $) / \operatorname{per}(A)$ of the bounded derived category $D^{b}(A$-mod) by the category of perfect complexes. In recent years, D. Orlov ([14]) rediscovered the notion of singularity categories in his study of $B$-branes on Landau-Ginzburg models in the framework of the Homological Mirror Symmetry Conjecture. The singularity category measures the homological singularity of an algebra in the sense that an algebra $A$ has finite global dimension if and only if its singularity category $D_{s g}(A)$ vanishes.

Two Artin algebras $A$ and $B$ are said to be singularly equivalent if there is a triangle equivalence between their singularity categories. In this case, the corresponding equivalence is called a singular equivalence between the two algebras. It is well known that derived equivalences can induce naturally singular equivalences. We recall that a derived equivalence between two algebras is a triangular equivalence between their bounded derived categories. J. Rickard ([15, Theorem 3.3]) proved that a tilting module $T$ over an algebra $A$ induces an equivalence between the derived category $D(A)$ and the derived category $D(B)$, where $B$ is the endomorphism algebra of $T$. Through this point we can get many examples of singular equivalences. Inspired by stable equivalences of Morita type introduced by M.

Supported by the National Natural Science Foundation of China (Grant No. 11771272).

Key words and phrases: singular equivalences, Auslander algebras, Morita rings, Gorensteinprojective modules. 
Broué ([3]), X.W. Chen and L.G. Sun ([10]) introduced a special singular equivalence between two $k$-algebras, which is called singularly equivalent of Morita type, as a generalization of stable equivalences of Morita type. Singularity categories and singular equivalences have drawn much attention. Their structural properties and construction were investigated among in e.g. [5], [6], [7], [8] and [9].

Recall that Morita rings are $2 \times 2$ matrix rings associated to Morita contexts $([2],[11])$. A particular case of interest is the Morita ring with bimodule homomorphisms zero. Gao and Psaroudakis [13] investigated its Gorenstein homological properties.

The aim of this article is to construct new classes of singular equivalences arising from Morita rings.

\section{Singular equivalences of Morita rings}

We first recall the definition of a singular equivalence of Morita type.

Definition 2.1. ([10, Definition 3.1]) Let $k$ be a field. Two finite-dimensional k-algebras $A$ and $B$ are singularly equivalent of Morita type if there exist an $A$ - $B$-bimodule ${ }_{A} M_{B}$ and a $B$ - $A$-bimodule ${ }_{B} N_{A}$ such that

(i) $M$ and $N$ are finitely generated projective as left and right modules;

(ii) $M \otimes_{B} N \cong A \oplus P$ as $A$ - $A$-bimodules for some finitely generated $A$ - $A$-bimodule $P$ with finite projective dimension, and $N \otimes_{A} M \cong B \oplus Q$ as $B$ - $B$-bimodules for some finitely generated $B$ - $B$-bimodule $Q$ with finite projective dimension.

Now we need recall the notion of a Gorenstein algebra and a Gorensteinprojective module. Let $A$ be a finite-dimensional $k$-algebra over a field $k . A$ is a $d$-Gorenstein algebra for some non-negative integer $d$ if the injective dimension of $A$ is finite and also equals $d$ as left and right $A$-modules. Denote by $A$-mod the category of finitely generated left $A$-modules, and by $A$-proj the full subcategory of finitely generated projective $A$-modules. An $A$-module $M$ in $A$-mod is called Gorenstein projective, if there exists an exact sequence $P^{\bullet}=\ldots \rightarrow P^{-1} \rightarrow P^{0} \stackrel{d^{0}}{\rightarrow} P^{1} \rightarrow \ldots$ in $A$-proj with $\operatorname{Hom}_{\Lambda}\left(P^{\bullet}, Q\right)$ exact for any $Q \in A$-proj, such that $M \cong \operatorname{ker} d^{0}$ (see [12]).

Lemma 2.2. Let $A$ and $B$ be two finite-dimensional $k$-algebras which are singularly equivalent of Morita type induced by bimodules $M$ and $N$. Then the following hold:

(1) The functors $M \otimes_{B}-: B-\bmod \rightarrow A$-mod and $N \otimes_{A}-: A$-mod $\rightarrow B$-mod are exact and take finitely generated projective modules to finitely generated projective modules. 
(2) Suppose that $A$ and $B$ are Gorenstein. Then the functors $M \otimes_{B}-$ and $N \otimes_{A}$ - induce a one-to-one correspondence between the indecomposable non-projective objects of $A$-Gproj and B-Gproj, where A-Gproj (resp. B-Gproj) denotes the category of finitely generated Gorenstein-projective A-modules (resp. B-modules).

Proof. (1) follows from the fact that ${ }_{A} M$ and ${ }_{B} N$ are finitely generated projective modules. (2) follows from [16, Proposition 3.7].

Lemma 2.3. Let $A$ and $B$ be two finite-dimensional Gorenstein $k$-algebras which are singularly equivalent of Morita type induced by bimodules $M$ and $N$. Let $P$ be a finitely generated $A$-A-bimodule satisfying Definition 2.1(ii) with the minimal projective resolution as $A$-A-bimodule

$$
0 \longrightarrow P_{n} \stackrel{d_{n}}{\longrightarrow} P_{n-1} \longrightarrow \ldots \longrightarrow P_{1} \stackrel{d_{1}}{\longrightarrow} P_{0} \longrightarrow{ }_{A} P_{A} \longrightarrow 0
$$

Let $C$ be a k-algebra and $V($ resp. $W)$ an $A$-C-bimodule (resp. $C$-A-bimodule) such that $V($ resp. $W)$ is a finitely generated non-projective Gorenstein-projective left (resp. right) A-module. Then we have the following:

(1) $P_{i} \otimes_{A} V=0$ for all $1 \leq i \leq n$, and $P_{0} \otimes_{A} V \cong P \otimes_{A} V$ as a left $A$-module.

(2) $W \otimes_{A} P_{i}=0$ for all $1 \leq i \leq n$, and $W \otimes_{A} P_{0} \cong W \otimes_{A} P$ as a right $A$-module.

(3) Let $\Lambda=\left(\begin{array}{cc}A & { }_{A} V_{C} \\ { }_{C} W_{A} & C\end{array}\right)$ be the Morita ring which is an Artin algebra such that $V \otimes_{C} W=0$ and $W \otimes_{A} V=0$. If $V$ and $W$ are indecomposable, then $P \otimes_{A} V=0$ and $W \otimes_{A} P=0$, and moreover, $P$ has finite projective dimension as a $\Lambda$ - $\Lambda$-bimodule.

Proof. (1). By assumption $P$ has finite projective dimension as a right $A$-module. Since $A$ is $d$-Gorenstein for some non-negative integer $d$, it follows that $\Omega^{d} P$ is Gorenstein-projective with finite projective dimension. So $\Omega^{d} P$ is either zero or projective. On the other hand, since ${ }_{A} V$ is Gorenstein-projective, it follows that there exists a left $A$-module $V_{1}$ such that $V \cong \Omega^{d} V_{1}$. Then for all $i \geq 1$,

$$
\operatorname{Tor}_{i}^{A}(P, V) \cong \operatorname{Tor}_{i}^{A}\left(P, \Omega^{d} V_{1}\right) \cong \operatorname{Tor}_{i}^{A}\left(\Omega^{d} P, V_{1}\right)=0
$$

So we obtain a long exact sequence of left $A$-modules

$$
\begin{aligned}
0 \longrightarrow & P_{n} \otimes_{A} V \stackrel{d_{n} \otimes \mathrm{Id}_{V}}{\longrightarrow} P_{n-1} \otimes_{A} V \longrightarrow \ldots \longrightarrow P_{1} \otimes_{A} V \stackrel{d_{1} \otimes \mathrm{Id}_{V}}{\longrightarrow} P_{0} \otimes_{A} V \longrightarrow P \otimes_{A} V \\
& \longrightarrow 0 \quad(* *)
\end{aligned}
$$

Since ${ }_{A}\left(P_{i} \otimes_{A} V\right)$ is projective for all $0 \leq i \leq n$ and $(*)$ is a minimal, then $(* *)$ is a minimal projective resolution of the left $A$-module $P \otimes_{A} V$. On the other hand, since $V$ and $M \otimes_{B} N \otimes_{A} V$ are both Gorenstein-projective left $A$-modules, and $M \otimes_{B} N \cong$ $A \oplus P$, it follows that $P \otimes_{A} V$ is also Gorenstein-projective. It follows that $M \otimes_{B}$ 
$N \otimes_{A} V \cong V \oplus P \otimes_{A} V$ and so $P \otimes_{A} V$ is a projective left $A$-module. Therefore, by the minimality of $(* *)$, we obtain that $P_{i} \otimes_{A} V=0$ for all $1 \leq i \leq n$, and $P_{0} \otimes_{A} V \cong P \otimes_{A} V$.

(2). By assumption $P$ has finite projective dimension as a left $A$-module. Since $A$ is $d$-Gorenstein for some non-negative integer $d$, it follows that $\Omega^{d} P$ is Gorensteinprojective with finite projective dimension. So $\Omega^{d} P$ is either zero or projective. On the other hand, since $W_{A}$ is Gorenstein-projective, it follows that there exists a right $A$-module $W_{1}$ such that $W \cong \Omega^{d} W_{1}$. Then for all $i \geq 1$,

$$
\operatorname{Tor}_{i}^{A}(W, P) \cong \operatorname{Tor}_{i}^{A}\left(\Omega^{d} W_{1}, P\right) \cong \operatorname{Tor}_{i}^{A}\left(W_{1}, \Omega^{d} P\right)=0
$$

So we obtain a long exact sequence of right $A$-modules

$$
\begin{aligned}
0 \longrightarrow W \otimes_{A} P_{n} \stackrel{\mathrm{Id}_{W} \otimes d_{n}}{\longrightarrow} W \otimes_{A} P_{n-1} \longrightarrow \ldots \longrightarrow W \otimes_{A} P_{1} \stackrel{\mathrm{Id}_{W} \otimes d_{1}}{\longrightarrow} W \otimes_{A} P_{0} \\
\longrightarrow W \otimes_{A} P \longrightarrow 0 \quad(* *)
\end{aligned}
$$

Since $\left(W \otimes{ }_{A} P_{i}\right)_{A}$ is projective for all $0 \leq i \leq n$ and $(*)$ is a minimal, then $(* *)$ is a minimal projective resolution of the right $A$-module $W \otimes_{A} P$. On the other hand, since $W$ and $W \otimes_{A} M \otimes_{B} N$ are both Gorenstein-projective right $A$-modules, and $M \otimes_{B} N \cong A \oplus P$, it follows that $W \otimes_{A} P$ is also Gorenstein-projective. It follows that $W \otimes_{A} M \otimes_{B} N \cong W \oplus W \otimes_{A} P$ and so $W \otimes_{A} P$ is a projective right $A$-module. Therefore, by the minimality of $(* *)$, we obtain that $W \otimes_{A} P_{i}=0$ for all $1 \leq i \leq n$, and $W \otimes_{A} P_{0} \cong W \otimes_{A} P$.

(3). Since $M \otimes_{B} N \cong A \oplus P$ as $A$ - $A$-bimodule, it follows that $M \otimes_{B} N \otimes_{A} V \cong V \oplus$ $\left(P \otimes_{A} V\right)$ as left $A$-modules. Then we have that $P \otimes_{A} V=0$. Since $P_{l}$ is a projective $A$-A-module for all $0 \leq l \leq n$, it follows that there exists a finite index set $I$ and pairs $\left(e_{i}, e_{j}\right)$ of idempotents of $A$ such that $P_{l}=\oplus_{(i, j) \in I} A e_{i} \otimes_{k} e_{j} A$. Since $P_{l} \otimes_{A} V=$ 0 , we have $e_{j} V \cong e_{j} A \otimes_{A} V=0$, and moreover, $e_{j} A \cong e_{j} A \oplus e_{j} V$ is a projective right $\Lambda$-module. On the other hand, $W \otimes_{A} M \otimes_{B} N \cong W \oplus\left(W \otimes_{A} P\right)$ as right $A$-modules. Then we have that $W \otimes_{A} P=0$. Since $P_{l}$ is a projective $A$ - $A$-module for all $0 \leq$ $l \leq n, P_{l}=\oplus_{(i, j) \in I} A e_{i} \otimes_{k} e_{j} A$. Since $W \otimes_{A} P_{l}=0$, we have $W e_{i} \cong W \otimes_{A} A e_{i}=0$, and moreover, $A e_{i} \cong A e_{i} \oplus W e_{i}$ is a projective left $\Lambda$-module. Thus we get that $A e_{i} \otimes_{k} e_{j} A$ is a projective $\Lambda$ - $\Lambda$-bimodule. This means that $P$ has finite projective dimension as a $\Lambda$ - $\Lambda$-bimodule.

Remark 2.4. Use the notation in Lemma 2.3. Let $\Gamma=\left(\begin{array}{cc}B & N \otimes_{A} V \\ W \otimes_{A} M & C\end{array}\right)$ be the Morita ring which is an Artin algebra. Since $N \otimes_{A} V$ and $W \otimes_{A} M$ are indecomposable non-projective Gorenstein-projective $B$-modules by Lemma 2.2 , we can adapt the proof of Lemma 2.3(3) to obtain that $Q$ is also a $\Gamma$ - $\Gamma$-bimodule with finite projective dimension such that $Q \otimes_{B} N \otimes_{A} V=0$ and $W \otimes_{A} M \otimes_{B} Q=0$. 
Theorem 2.5. Let $A$ and $B$ be Gorenstein $k$-algebras which are singularly equivalent of Morita type induced by bimodules $M$ and $N$. Let $C$ be a k-algebra. Let $V($ resp. $W)$ be an A-C-bimodule (resp. $C$-A-bimodule) such that $V($ resp. $W$ ) is a non-projective Gorenstein-projective left (resp. right) $A$-module and $V \otimes_{C}$ $W=0$ and $W \otimes_{A} V=0$. Let $\Lambda=\left(\begin{array}{cc}A & A V_{C} \\ C W_{A} & C\end{array}\right)$ and $\Gamma=\left(\begin{array}{cc}B & N \otimes_{A} V \\ W \otimes_{A} M & C\end{array}\right)$ be the Morita rings which are Artin algebras. Suppose that $\operatorname{End}_{B \otimes_{k} C^{\mathrm{op}}}\left(N \otimes_{A} V\right)=k \mathrm{Id}$ and $\operatorname{End}_{C \otimes_{k} B^{\mathrm{op}}}\left(W \otimes_{A} M\right)=k \mathrm{Id}$. Then $\Lambda$ and $\Gamma$ are singularly equivalent of Morita type.

Proof. By assumption, we have an $A$ - $A$-bimodule isomorphism $\rho=\left(\rho_{1}, \rho_{2}\right)$ : $M \otimes_{B} N \cong A \oplus P$ and a $B$-B-bimodule isomorphism $\sigma=\left(\sigma_{1}, \sigma_{2}\right): N \otimes_{A} M \cong B \oplus Q$, where $P$ and $Q$ have finite projective dimension. From the $A$ - $C$-bimodule isomorphism $M \otimes_{B} N \otimes_{A} V \cong V$, we have two $B$-C-bimodule isomorphisms $\operatorname{Id}_{N} \otimes \mu\left(\rho_{1} \otimes\right.$ $\left.\operatorname{Id}_{V}\right): N \otimes_{A} M \otimes_{B} N \otimes_{A} V \cong N \otimes_{A} V$ and $\mu^{\prime}\left(\sigma_{1} \otimes \operatorname{Id}_{N \otimes_{A} V}\right): N \otimes_{A} M \otimes_{B} N \otimes_{A} V \cong N \otimes_{A}$ $V$, where $\mu: A \otimes_{A} V \rightarrow V$ and $\mu^{\prime}: B \otimes_{B}\left(N \otimes_{A} V\right) \rightarrow N \otimes_{A} V$ are the multiplication maps. Since $\operatorname{End}_{B \otimes_{k} C^{\mathrm{op}}}\left(N \otimes_{A} V\right)=k \mathrm{Id}$, there exists a non-zero element $k_{0} \in k$ such that $\operatorname{Id}_{N} \otimes \mu\left(\rho_{1} \otimes \operatorname{Id}_{V}\right)=k_{0}\left(\mu^{\prime}\left(\sigma_{1} \otimes \operatorname{Id}_{N \otimes_{A} V}\right)\right)$. Without loss of generality, we may assume that $k_{0}=1$. On the other hand, from the $C$-A-bimodule isomorphism $W \otimes_{A} M \otimes_{B} N \cong W$, we have two $A$-C-bimodule isomorphisms $\mu^{\prime \prime}\left(\operatorname{Id}_{W} \otimes \rho_{1}\right) \otimes \operatorname{Id}_{M}$ : $W \otimes_{A} M \otimes_{B} N \otimes_{A} M \cong W \otimes_{A} M$ and $\mu^{\prime \prime \prime}\left(\operatorname{Id}_{W \otimes_{A} M} \otimes \sigma_{1}\right): W \otimes_{A} M \otimes_{B} N \otimes_{A} M \cong W \otimes_{A}$ $M$, where $\mu^{\prime \prime}: W \otimes_{A} A \rightarrow W$ and $\mu^{\prime \prime \prime}:\left(W \otimes_{A} M\right) \otimes_{B} B \rightarrow W \otimes_{A} M$ are the multiplication maps. Since $\operatorname{End}_{C \otimes_{k} B^{\text {op }}}\left(W \otimes_{A} M\right)=k \mathrm{Id}$, there exists a non-zero element $k_{0}^{\prime} \in k$ such that $\mu^{\prime \prime}\left(\operatorname{Id}_{W} \otimes \rho_{1}\right) \otimes \operatorname{Id}_{M}=k_{0}^{\prime}\left(\mu^{\prime \prime \prime}\left(\operatorname{Id}_{W \otimes_{A} M} \otimes \sigma_{1}\right)\right)$. Without loss of generality, we may assume that $k_{0}^{\prime}=1$.

Recall that each finitely generated $\Lambda$-module $X$ can be described as a tuple $X=\left(X_{0}, X_{\omega}, f, g\right)$, where $X_{0}$ is in $A$-mod, $X_{\omega}$ is in $C$-mod, and $f: V \otimes_{C} X_{\omega} \rightarrow X_{0}$ is an $A$-homomorphism, $g: W \otimes_{A} X_{0} \rightarrow X_{\omega}$ is a $C$-homomorphism, by the way that $X=$ $X_{0} \oplus X_{\omega}$ with $\Lambda$-module structure given by $\left(\begin{array}{cc}a & v \\ w & c\end{array}\right)(x, y)=(a x+f(v \otimes y), g(w \otimes x)+$ $c y)$. Now given a $\Lambda$-module $X=\left(X_{0}, X_{\omega}, f, g\right)$, we put $F(X):=\left(N \otimes_{A} X_{0}, X_{\omega}, f\left(\mu^{\prime \prime} \otimes\right.\right.$ $\left.\left.\operatorname{Id}_{X_{0}}\right)\left(\operatorname{Id}_{W} \otimes \rho_{1} \otimes \operatorname{Id}_{X_{0}}\right), \operatorname{Id}_{N} \otimes g\right)$. Then $F: \Lambda$-mod $\rightarrow \Gamma$-mod is a well-defined exact functor preserving finitely generated projective modules. By Watt's Theorem (e.g. [17, Theorem 3.3.16]), $F \cong_{\Gamma} F(\Lambda) \otimes_{\Lambda}-$. Now let us define the functor $G: \Gamma-\bmod \rightarrow$ $\Lambda$-mod.

Recall that each finitely generated $\Gamma$-module $Y$ can be described as a tuple $Y=(U, T, s, t)$, where $U$ is in $B$-mod, $T$ is in $C$-mod, and $s: N \otimes_{A} V \otimes_{C} T \rightarrow U$ is a $B$-homomorphism, $t: W \otimes_{A} M \otimes_{C} U \rightarrow T$ is a $C$-homomorphism, by the way that $Y=U \oplus T$ with $\Gamma$-module structure given by $\left(\begin{array}{cc}b & n \otimes v \\ w \otimes m & c\end{array}\right)(u, p)=(b u+s(n \otimes v \otimes$ $p), t(w \otimes m \otimes u)+c p)$. Now for $(U, T, s, t) \in \Gamma$-mod with the $C$-morphism $s: W \otimes_{A}$ 
$M \otimes_{B} U \rightarrow T$ and the $B$-morphism $t: N \otimes_{A} V \otimes_{C} T \rightarrow U$, we define

$$
G(U, T, s, t):=\left(M \otimes_{B} U, T, s,\left(\operatorname{Id}_{M} \otimes t\right)\left(\left(\mu\left(\rho_{1} \otimes \operatorname{Id}_{V}\right)\right)^{-1} \otimes \operatorname{Id}_{T}\right)\right.
$$

Then $G$ is a well-defined exact functor preserving finitely generated projective modules. By Watts' Theorem (e.g. [17, Theorem 3.3.16]), $G \cong{ }_{\Lambda} G(\Gamma) \otimes_{\Gamma}-$. Denote by $\mu^{\prime \prime \prime \prime}:\left(N \otimes_{A} V\right) \otimes_{C} C \rightarrow N \otimes_{A} V$ and $\mu^{\prime \prime \prime \prime \prime \prime}: V \otimes_{C} C \rightarrow V$ the multiplication maps. Since there are the $\Lambda$ - $\Lambda$-bimodule isomorphisms

$$
\begin{aligned}
G(\Gamma) \otimes_{\Gamma} F(\Lambda) & \cong G(F(\Lambda))=G\left(F\left(\left(A, W, \mu^{\prime \prime}, 0\right) \oplus\left(V, C, 0, \mu^{\prime \prime \prime \prime}\right)\right)\right) \\
& =G\left(\left(N \otimes_{A} A, W, \mu^{\prime \prime}\left(\mu^{\prime \prime} \otimes \operatorname{Id}_{A}\right)\left(\operatorname{Id}_{W} \otimes \rho_{1} \otimes \operatorname{Id}_{A}\right), 0\right)\right. \\
& \left.\oplus\left(N \otimes_{A} V, C, 0, \operatorname{Id}_{N} \otimes \mu^{\prime \prime \prime \prime \prime \prime}\right)\right) \\
& =\left(M \otimes_{B} N \otimes_{A} A, W, \mu^{\prime \prime}\left(\mu^{\prime \prime} \otimes \operatorname{Id}_{A}\right)\left(\operatorname{Id}_{W} \otimes \rho_{1} \otimes \operatorname{Id}_{A}\right), 0\right) \\
& \oplus\left(M \otimes_{B} N \otimes_{A} V, C, 0,\left(\mu\left(\rho_{1} \otimes \operatorname{Id}_{V}\right)\right)^{-1} \mu^{\prime \prime \prime \prime \prime}\right) \\
& \cong \Lambda \oplus P
\end{aligned}
$$

and the $\Gamma$ - $\Gamma$-bimodule isomorphisms

$$
\begin{aligned}
F(\Lambda) \otimes_{\Lambda} G(\Gamma) & \cong F(G(\Gamma))=F\left(G\left(\left(B, W \otimes_{B} M, \mu^{\prime \prime \prime}, 0\right) \oplus\left(N \otimes_{A} V, C, 0, \mu^{\prime \prime \prime \prime}\right)\right)\right) \\
& =F\left(\left(M \otimes_{B} B, W \otimes_{B} M, \mu^{\prime \prime \prime}, 0\right)\right. \\
& \oplus\left(M \otimes_{B} N \otimes_{A} V, C, 0,\left(\mu\left(\rho_{1} \otimes \operatorname{Id}_{V}\right)\right)^{-1} \mu^{\prime \prime \prime \prime \prime}\right) \\
& =\left(N \otimes_{A} M \otimes_{B} B, W \otimes_{B} M, \mu^{\prime \prime \prime}\left(\mu^{\prime \prime} \otimes \operatorname{Id}_{B}\right)\left(\operatorname{Id}_{W} \otimes \rho_{1} \otimes \operatorname{Id}_{B}\right), 0\right) \\
& \oplus\left(N \otimes_{A} M \otimes_{B} N \otimes_{A} V, C, 0,\left(\operatorname{Id}_{N} \otimes\left(\mu\left(\rho_{1} \otimes \operatorname{Id}_{V}\right)\right)^{-1}\right) \mu^{\prime \prime \prime \prime}\right) \\
& \cong \Gamma \oplus Q
\end{aligned}
$$

it follows from Lemma 2.3 and Remark 2.4 that $\Lambda$ and $\Gamma$ are singularly equivalent of Morita type.

Corollary 2.6. Let $A$ and $B$ be Gorenstein k-algebras which are singularly equivalent of Morita type induced by bimodules $M$ and $N$. Let $C$ be a k-algebra and $V$ an $A$-C-bimodule such that $V$ is an indecomposable non-projective Gorensteinprojective left $A$-module such that $\operatorname{End}_{B \otimes_{k} C^{o p}}\left(N \otimes_{A} V\right)=k \operatorname{Id}$. Let $\Lambda=\left(\begin{array}{cc}A_{A} V_{C} \\ 0 & C\end{array}\right)$ and $\Gamma=\left(\begin{array}{cc}B & N \otimes_{A} V \\ 0 & C\end{array}\right)$. Then $\Lambda$ and $\Gamma$ are singularly equivalent of Morita type.

Hochschild homology was introduced by Hochschild as a tool for studying the structure of associative algebras. Hochschild homology of a finite dimensional algebra has a very rich structure. It has a cup of product making it into a graded commutative algebra; it also has a Lie bracket of degree -1 , making it into a graded 
Lie algebra. Zhou-Zimmermann ([18]) proved that a singular equivalence of Morita type between finite dimensional $k$-algebras preserve the Hochschild homology group. We recall the notion of the Hochschild homology group.

Let $A$ be an Artin algebra and $A^{e}=A \otimes_{k} A^{\text {op }}$ be the enveloping algebra. Let $M$ be an $A$-bimodule. Recall that the Hochschild homology group of $A$ with coefficients in $M$ is defined as $\operatorname{HH}_{n}(A, M)=\operatorname{Tor}_{n}^{A^{e}}(A, M)$.

Corollary 2.7. Let $A$ and $B$ be Gorenstein $k$-algebras which are singularly equivalent of Morita type. Suppose that $C, W, V, \Lambda$ and $\Gamma$ be as above in Theorem 2.5. Then $\Lambda$ and $\Gamma$ have isomorphic Hochschild homology groups for each $n>0$,

$$
\mathrm{HH}_{n}(\Lambda) \cong \mathrm{HH}_{n}(\Gamma)
$$

Proof. By Theorem 2.5, we know that $\Lambda$ and $\Gamma$ are singularly equivalent of the Morita type. Thus by [18, Theorem 4.1] there are isomorphisms of the Hochschild homology groups for each $n>0$,

$$
\mathrm{HH}_{n}(\Lambda) \cong \mathrm{HH}_{n}(\Gamma)
$$

\section{References}

1. Auslander, M., The Representation Dimension of Artin Algebras, Queen Mary College Mathematics Notes, American Mathematical Society, Providence, 1971. Republished in Selected works of Maurice Auslander, 1999. MR1674397

2. BAss, H., The Morita theorems, Mimeographed notes, 1962.

3. Broué, M., Equivalences of blocks of group algebras, in Finite Dimensional Algebras and Related Topics, pp. 1-26, Kluwer, 1994. MR1308978

4. Buchweitz, R. O., Maximal Cohen-Macaulay modules and Tate-cohomology over Gorenstein rings, Preprint, 1987. Available in: http://hdl.handle.net/ $1807 / 16682$.

5. Chen, X. W., Singularity categories, Schur functors and triangular matrix rings, Algebr. Represent. Theory 12 (2009), 181-191. MR2501179

6. Chen, X. W., Singular equivalences induced by homological epimorphisms, Proc. Amer. Math. Soc. 142 (2014), 2633-2640. MR3209319

7. Chen, X. W., Singular equivalences of trivial extensions, Comm. Algebra 44 (2016), 1961-1970. MR3490659

8. Chen, X. W., Geng, S. F. and Lu, M., The singularity categories of the Clustertilted algebras of Dynkin type, Algebr. Represent. Theory 18 (2015), 531554. MR3336352

9. Chen, X. W. and Lu, M., Singularity categories of skewed-gentle algebras, Colloq. Math. 141 (2015), 183-198. MR3404262

10. Chen, X. W. and Sun, L. G., Singular equivalences of Morita type, Preprint, 2012. MR3373371 
11. Cohn, P. M., Morita Equivalence and Duality, Queen Mary College Math. Notes, 1966. MR0258885

12. Enochs, E. E. and Jenda, O. M. G., Relative Homological Algebra, De Gruyter Exp. Math. 30, Walter de Gruyter Co., 2000. MR1753146

13. GaO, N. and Psaroudakis, C., Gorenstein homological aspects of monomorphism categories via Morita rings, Algebr. Represent. Theory 20 (2017), 487529. MR3638357

14. OrLov, D., Triangulated categories of singularities and D-branes in Landau-Ginzburg models, Proc. Steklov Inst. Math. 246 (2004), 227-248. MR2101296

15. Rickard, J., Morita theory for derived categories, J. Lond. Math. Soc. 39 (1989), 436-456. MR1002456

16. Skartsæterhagen, $\varnothing$., Singular equivalence and the (Fg) condition, J. Algebra 452 (2016), 66-93. MR3461056

17. Zimmermann, A., Representation Theory: A Homological Algebra Point of View, Algebra and Applications 19, Springer, 2014. MR3289041

18. Zhou, G. D. and Zimmermann, A., On singular equivalences of Morita type, J. Algebra 385 (2013), 64-79. MR3049562

Nan Gao

Department of Mathematics

Shanghai University

Shanghai CN-200444

PR China

nangao@shu.edu.cn

Received December 27, 2018

in revised form July 4, 2019
Wen-Hui Zhao

Department of Mathematics

Shanghai University

Shanghai CN-200444

PR China

huihui0524@i.shu.edu.cn 